\title{
INVARIANCE OF THE JACOBIAN NEWTON DIAGRAM
}

\author{
JANUSZ GWOŹDZIEWICZ
}

Abstract. We prove that the jacobian Newton diagram of the holomorphic mapping $(f, g):\left(\mathbb{C}^{2}, 0\right) \rightarrow\left(\mathbb{C}^{2}, 0\right)$ depends only on the equisingularity class of the pair of curves $f=0$ and $g=0$.

\section{Introduction}

Write $\mathbb{R}_{+}=\{x \in \mathbb{R}: x \geq 0\}$. The Newton diagram $\Delta_{h}$ of a power series $h(x, y)=$ $\sum_{i, j} c_{i j} x^{i} y^{j}$ is by definition the convex hull of the union

$$
\bigcup_{\left\{(i, j): c_{i j} \neq 0\right\}}\left\{(i, j)+\mathbb{R}_{+}^{2}\right\}
$$

Example 1.1. The Newton diagram of $h(x, y)=y^{5}+2 x y^{3}-x^{3} y^{2}+3 x^{4} y$ is drawn in the figure. Black dots are the points of the first quadrant $\mathbb{R}_{+}^{2}$ corresponding to nonzero monomials of the series $h$.

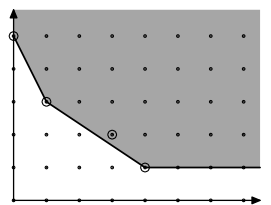

Let $\phi:\left(\mathbb{C}^{2}, 0\right) \rightarrow\left(\mathbb{C}^{2}, 0\right), \phi^{-1}(0,0)=\{(0,0)\}$ be the germ of a holomorphic mapping given by $(x, y)=(f(u, v), g(u, v))$. Let jac $\phi=\frac{\partial f}{\partial u} \frac{\partial g}{\partial v}-\frac{\partial f}{\partial v} \frac{\partial g}{\partial u}$ be the usual jacobian determinant. The direct image of the curve germ jac $\phi=0$ by $\phi$ is called the discriminant curve of $\phi$ (see [2]). If $D(x, y)=0$ is an analytic equation of the discriminant curve then the Newton diagram of $D$ is called the jacobian Newton diagram of $(f, g)$. We will write $\mathcal{N}_{J}(f, g)$ for the jacobian Newton diagram.

Let $h=h(u, v) \in \mathbb{C}\{u, v\}, h(0,0)=0$ be a convergent power series and let $h=$ $h_{1}^{m_{1}} \cdots h_{s}^{m_{s}}$ be a factorization of $h$ in the ring $\mathbb{C}\{u, v\}$ with $h_{i}$ irreducible and pairwise co-prime. Then every curve germ $h_{i}=0$ is called a branch of the curve $h=0$ and $m_{i}$ is called the multiplicity of $h_{i}=0$ in $h=0$.

Definition 1.1. Let $\xi, \xi^{\prime}, \nu, \nu^{\prime}$ be germs of analytic curves in $\left(\mathbb{C}^{2}, 0\right)$. We say that the pairs of curves $\xi, \nu$ and $\xi^{\prime}, \nu^{\prime}$ are equisingular if there exists a homeomorphism $\Psi:\left(\mathbb{C}^{2}, 0\right) \rightarrow\left(\mathbb{C}^{2}, 0\right)$ preserving for each curve the multiplicity in it of each of its branches such that $\Psi(\xi)=\xi^{\prime}$ and $\Psi(\nu)=\nu^{\prime}$.

Received by the editors November 21, 2011. 


\section{Main result}

Theorem 2.1. Let $(f, g):\left(\mathbb{C}^{2}, 0\right) \rightarrow\left(\mathbb{C}^{2}, 0\right),(f, g)^{-1}(0,0)=\{(0,0)\}$ be the germ of a holomorphic mapping. Then the jacobian Newton diagram $\mathcal{N}_{J}(f, g)$ depends only on the equisingularity class of the pair of curves $f=0$ and $g=0$.

The proof is in the last section.

We give a survey of results related with Theorem 2.1. We need a few notions which will be used only in this section to explain connection between certain analytic factorizations of $\operatorname{jac}(f, g)$ and the jacobian Newton diagram $\mathcal{N}_{J}(f, g)$.

The Minkowski sum of Newton diagrams $\Delta_{1}$ and $\Delta_{2}$ is by definition $\Delta_{1}+\Delta_{2}=$ $\left\{p+q: p \in \Delta_{1}, q \in \Delta_{2}\right\}$. The set of Newton diagrams is a semi-group with respect to Minkowski sum and the generators of this semi-group are elementary Newton diagrams illustrated in Figure 1.

The inclination of the elementary Newton diagram $\left\{\frac{a}{b}\right\}$ is the quotient $\frac{a}{b}$ (by convention $\frac{\infty}{b}=\infty$ and $\left.\frac{a}{\infty}=0\right)$. For an arbitrary Newton diagram $\Delta$ represented as a sum of elementary Newton diagrams let us denote $I(\Delta)$ the set of inclinations of elementary Newton diagrams of the sum. It is easy to check that $I(\Delta)$ does not depend on the choice of a representation. Coming back to Example 1.1 the Newton diagram $\Delta_{h}$ is the $\operatorname{sum}\left\{\frac{1}{2}\right\}+\left\{\frac{3}{2}\right\}+\left\{\frac{\infty}{1}\right\}$ and $I\left(\Delta_{h}\right)=\{1 / 2,3 / 2, \infty\}$.

If $h$ is any irreducible factor of $\operatorname{jac}(f, g)$ then $q(h)=\frac{i_{0}(g, h)}{i_{0}(f, h)}$, where $i_{0}(\cdot, \cdot)$ stands for the intersection multiplicity, is called the jacobian quotient of $(f, g)$.

Definition 2.1. Let jac $(f, g)=J_{1} \cdots J_{n}$ be an analytic factorization of the jacobian.

We will call $J_{1} \cdots J_{n}$ a Hironaka factorization if for every $J_{i}(1 \leq i \leq n)$ the jacobian quotient $q(h)$ is constant for all irreducible factors $h$ of $J_{i}$.

The Hironaka factorization $J_{1} \cdots J_{n}$ will be called minimal if jacobian quotients of irreducible factors of $J_{l}$ and $J_{k}$ are different for $1 \leq l<k \leq n$.

Let jac $(f, g)=h_{1} \cdots h_{n}$ be the factorization of the jacobian into irreducible factors. It is easy to check (see [13]) that

$$
\mathcal{N}_{J}(f, g)=\sum_{i=1}^{n}\left\{\frac{i_{0}\left(g, h_{i}\right)}{\overline{i_{0}\left(f, h_{i}\right)}}\right\} .
$$

It follows directly from the above formula that

- the set of jacobian quotients of $(f, g)$ is the set of inclinations of $\mathcal{N}_{J}(f, g)$,

- if $J_{1} \cdots J_{r}$ is a Hironaka factorization of $\operatorname{jac}(f, g)$ then

$$
\mathcal{N}_{J}(f, g)=\sum_{i=1}^{r}\left\{\frac{i_{0}\left(g, J_{i}\right)}{\overline{i_{0}\left(f, J_{i}\right)}}\right\},
$$
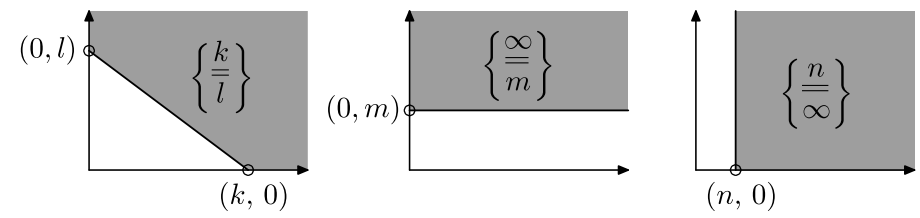

FiguRE 1. Elementary Newton diagrams 
- if $\mathcal{N}_{J}(f, g)=\sum_{i=1}^{s}\left\{\frac{a_{i}}{b_{i}}\right\}$ with inclinations $\frac{a_{i}}{b_{i}}$ pairwise different then $\operatorname{jac}(f, g)$ has the minimal Hironaka factorization $J_{1} \cdots J_{s}$ such that $i_{0}\left(g, J_{i}\right)=a_{i}$ and $i_{0}\left(f, J_{i}\right)=b_{i}$ for $i=1, \ldots, s$.

Let us consider a germ of a holomorphic mapping $(l, f):\left(\mathbb{C}^{2}, 0\right) \rightarrow\left(\mathbb{C}^{2}, 0\right)$ such that $f=0$ is a curve germ without multiple branches and $l=0$ is a generic smooth curve (here generic means that the curve $l=0$ is not tangent to any branch of the curve $f=0)$. Under these assumptions $\operatorname{jac}(l, f)=0$ is called the polar curve of $f$ and jacobian quotients of $(l, f)$ are called polar quotients. A survey of recent results concerning polar curves is in [6].

Merle in [10] obtained the minimal Hironaka decomposition of the polar curve of the irreducible curve germ $f=0$. Merle's result is rewritten in [13] as a formula for the jacobian Newton diagram of $(l, f)$ (see also [6], Theorem 4.1).

In [7], Kuo and Lu described the contact orders of Newton-Puiseux roots of the partial derivative $f_{x}^{\prime}(x, y)=0$ with the Newton-Puiseux roots of $f(x, y)=0$. They constructed the tree model $T(f)$ which encodes these contact orders. Using the KuoLu tree $T(f)$ one can compute all polar quotients of $(y, f)$. One can also give a formula for the jacobian Newton diagram of $(y, f)$ in terms of $T(f)$ (see the last line before Example 5.2 in [5]).

In [4], the author studied the polar curve of a many-branched curve $f=0$. He introduced a new type of tree $E(f)$ called now the Eggers tree of $f$. Eggers found the Hironaka factorization of the jacobian jac $(l, f)$ such that the factors are indexed by vertices of $E(f)$. He also computed the intersection multiplicities of every factor with $l$ and $f$. Since the Eggers tree $E(f)$ depends only on the equisingularity class of $f$, Theorem 2.1 in this particular case follows from [4].

The papers [7] and [4] provide methods of computing $\mathcal{N}_{J}(l, f)$ using invariants of equisingularity of $f$. Another way to obtain Theorem 2.1 in the polar case is to use a deformation argument. Teissier proved in [14] that for every $\mu^{*}$-constant family of hypersurfaces with isolated singularities, the jacobian Newton diagram is constant. Since every two plane analytic curves of the same equisingularity type can be joined by a $\mu^{*}$-constant family of plane curves (see [2], Proposition 5.2 for a direct construction of such a family) we get another proof of Theorem 2.1 in the polar case.

Consider now a general case of a holomorphic mapping germ $(f, g):\left(\mathbb{C}^{2}, 0\right) \rightarrow$ $\left(\mathbb{C}^{2}, 0\right)$, where $(f, g)^{-1}(0,0)=\{(0,0)\}$.

In [8], the authors additionally assumed that the curve $f g=0$ has no multiple components. They defined the equivalence relation between vertices of the Kuo-Lu tree $T(f g)$. Then they obtained the Hironaka factorization of jac $(f, g)$ such that the factors are indexed by equivalence classes of this relation. However, as Section 5 of [8] shows, the equisingularity class of the pair $f=0, g=0$ does not determine the intersection multiplicities of some factors with $f$ and $g$. Hence, Theorem 4.1 does not follow from [8].

In [9] and [11], the authors resolved singularities of the curve $f g=0$. Then they distinguished some subsets of the exceptional divisor called rupture zones and associated a factor of the jacobian jac $(f, g)$ with every rupture zone. Maugendre [9] found the set of jacobian quotients using topological methods (see also [3] for an algebraic proof) and Michel [11] completed the work computing the intersection multiplicities of every factor with $f$ and $g$. Since the decomposition of the jacobian obtained by Michel is a 
Hironaka factorization, Theorem 2.1 follows from [11]. However, the proof presented in this article is much simpler as it uses only Theorem 3.1 and the following formula.

Theorem 2.2 ([2] Theorem 3.2). Let $(f, g):\left(\mathbb{C}^{2}, 0\right) \rightarrow\left(\mathbb{C}^{2}, 0\right)$ be a germ of a holomorphic mapping such that $(f, g)^{-1}(0,0)=\{(0,0)\}$. Let $D(x, y)=0$ be the discriminant curve of $(f, g)$. Take any curve germ $h(x, y)=0$ and let $H(u, v)=$ $h(f(u, v), g(u, v))$. Then

$$
\mu_{0}(H)-1=i_{0}(f, g)\left[\mu_{0}(h)-1\right]+i_{0}(h, D),
$$

where $\mu_{0}(h)$ denotes the Milnor number of the curve $h=0$ at zero.

\section{Invariance of a generic curve of the pencil}

Theorem 3.1. Let $(f, g):\left(\mathbb{C}^{2}, 0\right) \rightarrow\left(\mathbb{C}^{2}, 0\right),(f, g)^{-1}(0,0)=\{(0,0)\}$ be the germ of a holomorphic mapping. Then for all $t \in \mathbb{C}$ but a finite number the equisingularity class of the curve $f(x, y)-\operatorname{tg}(x, y)=0$ depends only on the equisingularity class of the pair of curves $f=0$ and $g=0$.

Proof. Our main reference is Chapter III of [12]. Let $R: M \rightarrow\left(\mathbb{C}^{2}, 0\right)$ be the minimal good resolution of singularities of the curve $f g=0$. The set $R^{-1}(\{f g=0\})$ can be written as the union of irreducible components $E_{1} \cup \cdots \cup E_{n} \cup E_{n+1} \cup \cdots \cup E_{m}$, where $E=E_{1} \cup \cdots \cup E_{n}$ is the exceptional divisor $R^{-1}(0)$ and $E_{n+1}, \ldots, E_{m}$ are noncompact curves corresponding with branches of the curve $f g=0$. Put $\tilde{f}=f \circ R$, $\tilde{g}=g \circ R$ and let $a_{i}=$ order of $\tilde{f}$ along $E_{i}, b_{i}=$ order of $\tilde{g}$ along $E_{i}$ for $i=1, \ldots, m$. Then, after renumbering $E_{1}, \ldots, E_{m}$ if necessary, the total dual resolution graph as well as the numbers $a_{i}$ and $b_{i}$ for $i=1, \ldots, m$ depend only on the equisingularity class of the pair of curves $f=0$ and $g=0$.

Consider the meromorphic function $\tilde{f} / \tilde{g}: M \backslash E \rightarrow \mathbb{C} \cup\{\infty\}$. We will check that this function extends analytically to the whole $M$ with the exception of a finite number of points. Let - - denotes any germ of a holomorphic function $u(x, y)$ such that $u(0,0) \neq 0$.

First take $P \in E_{i}(1 \leq i \leq n)$ which is not an intersection point with another component $E_{j}$ for $1 \leq j \leq m$. Choose a local analytical coordinate system $(x, y)$ centered at $P$ such that $E_{i}$ has the equation $x=0$. In these coordinates $\tilde{f}=-x^{a_{i}}$ and $\tilde{g}=-x^{b_{i}}$. We get $\tilde{f} / \tilde{g}=-x^{a_{i}-b_{i}}$.

Now take the intersection point $P$ of $E_{i}$ with another component $E_{j}$. Choose a local analytical coordinate system $(x, y)$ centered at $P$ such that $E_{i}$ has the equation $x=0$ and $E_{j}$ has the equation $y=0$. In these coordinates $\tilde{f}=-x^{a_{i}} y^{a_{j}}$ and $\tilde{g}=-x^{b_{i}} y^{b_{j}}$. We get $\tilde{f} / \tilde{g}=-x^{a_{i}-b_{i}} y^{a_{j}-b_{j}}$.

Let $H$ be an analytic extension of $\tilde{f} / \tilde{g}$. Divide the set $\left\{E_{1}, \ldots, E_{m}\right\}$ into three subsets $A_{+}=\left\{E_{i}: a_{i}-b_{i}>0\right\}, A_{0}=\left\{E_{i}: a_{i}-b_{i}=0\right\}$ and $A_{-}=\left\{E_{i}: a_{i}-b_{i}<\right.$ $0\}$. It follows from the above description of $\tilde{f} / \tilde{g}$ near $E$ that $H$ is not defined only at the intersection points of components from $A_{+}$with components from $A_{-}$.

Let $E_{i} \in A_{0}$. Consider the restriction $\left.H\right|_{E_{i}}$ of the meromorphic function $H$ to $E_{i}$. Then $P \in E_{i}$ is a zero of $\left.H\right|_{E_{i}}$ if and only if $\{P\}=E_{i} \cap E_{j}$ for some $E_{j} \in A_{+}$. Moreover, $\left.\operatorname{ord}_{P} H\right|_{E_{i}}=a_{j}-b_{j}$. Hence the topological degree of $\left.H\right|_{E_{i}}$ is the number $d_{i}=\sum\left(a_{j}-b_{j}\right)$ where the sum runs over all $j$ such that $E_{j} \in A_{+}$and the intersection $E_{i} \cap E_{j}$ is nonempty. 
Choose a nonzero complex number $t$ which is different from

- any critical value of meromorphic functions $\left.H\right|_{E_{i}}$ where $E_{i} \in A_{0}$,

- any value $H(P)$ where $P$ is the intersection point of some $E_{i} \in A_{0}$ with some $E_{j}, j \neq i$.

Let $\Gamma$ be the proper preimage of the curve $f-t g=0$. The curve $\Gamma$ has an equation $H=t$ at every point where $H$ is well defined. Hence $\Gamma$ intersects transversally every $E_{i} \in A_{0}$ at $d_{i}$ points and none of these points belong to $\bigcup_{j \neq i} E_{j}$.

Now we compute the equation of $\Gamma$ near points where $H$ is not defined. Take $E_{i} \in A_{+}, E_{j} \in A_{-}$with nonempty intersection and denote $P_{i, j}$ their intersection point. There exists a local analytical coordinate system $(x, y)$ centered at $P_{i, j}$ such that $E_{i}$ has the equation $x=0$ and $E_{j}$ has the equation $y=0$. In these coordinates $\tilde{f}-t \tilde{g}=-x^{a_{i}} y^{a_{j}}-t-x^{b_{i}} y^{b_{j}}=-x^{b_{i}} y^{a_{j}}\left(-x^{a_{i}-b_{i}}-t y^{b_{j}-a_{j}}\right)$, hence $\Gamma$ has the equation $-x^{a_{i}-b_{i}}-t y^{b_{j}-a_{j}}=0$.

We want to resolve singularities of the curve $\Gamma$ to obtain a good (not necessarily minimal) resolution of singularities of $f-t g=0$. Every function $h_{i, j}(x, y)=$ $-x^{a_{i}-b_{i}}-t y^{b_{j}-a_{j}}$ is nondegenerate with the Newton diagram $\left\{\frac{a_{i}-b_{i}}{\overline{b_{j}-a_{j}}}\right\}$. Hence by Theorem 4.3 of [12] there exists a canonical toric resolution of $h_{i, j}(x, y)=0$ at the origin, that is the resolution of $\Gamma$ at $P_{i, j}$, which depends only on the Newton diagram of $h_{i, j}$. Applying such a toric resolution at every point $P_{i, j}$ described above we obtain a good resolution of $f-t g=0$. Moreover, the total dual resolution graph of this resolution depends only on the total dual resolution graph of $R$ and on the numbers $a_{i}$ and $b_{i}$ for $i=1, \ldots, m$.

Since the total dual resolution graph of the plane curve singularity determines its equisingularity class (see [1], Chapter 8.4, Proposition 20) the proof is finished.

\section{Proof of the main result}

For every Newton diagram $\Delta$ and for every $\vec{v}=\left(v_{1}, v_{2}\right)$, where $v_{1}>0, v_{2}>0$ we define

$$
l(\vec{v}, \Delta)=\min \left\{v_{1} i+v_{2} j:(i, j) \in \Delta\right\} .
$$

Lemma 4.1. Let $\vec{v}=(m, n)$, where $n, m$ are co-prime positive integers. Then for generic $t \in \mathbb{C}$

$$
l\left(\vec{v}, \mathcal{N}_{J}(f, g)\right)=\mu_{0}\left(f^{n}-t g^{m}\right)-i_{0}(f, g)[(m-1)(n-1)-1]-1 .
$$

Proof. Let $D=0$, where $D(x, y)=\sum c_{i j} x^{i} y^{j}$, be the equation of the discriminant curve of $\phi=(f, g):\left(\mathbb{C}^{2}, 0\right) \rightarrow\left(\mathbb{C}^{2}, 0\right)$. Take a curve $x^{n}-t y^{m}=0$.

Claim. For generic $t \in \mathbb{C}$ we have $i_{0}\left(x^{n}-t y^{m}, D\right)=l\left(\vec{v}, \mathcal{N}_{J}(f, g)\right)$.

Let $\tau=\sqrt[n]{t}$. Then $x=\tau s^{m}, y=s^{n}$ is a parameterization of the branch $x^{n}-t y^{m}=$ 0 . By the classical formula for the intersection multiplicity

$$
i_{0}\left(x^{n}-t y^{m}, D\right)=\operatorname{ord}_{s} D\left(\tau s^{m}, s^{n}\right)=\operatorname{ord}_{s} \sum c_{i j} \tau^{i} s^{m i+n j}=l\left(\vec{v}, \mathcal{N}_{J}(f, g)\right)
$$

provided $\tau$ is sufficiently general so that the sum $\sum_{m i+n j=l\left(\vec{v}, \mathcal{N}_{J}(f, g)\right)} c_{i j} \tau^{i}$ is nonzero. The Claim is proved. 
The pull-back of the curve $x^{n}-t y^{m}=0$ by $\phi$ has an equation $f^{n}-t g^{m}=0$. Thus by Theorem 2.2 we have

$$
\mu_{0}\left(f^{n}-t g^{m}\right)-1=i_{0}(f, g)\left[\mu_{0}\left(x^{n}-t y^{m}\right)-1\right]+i_{0}\left(x^{n}-t y^{m}, D\right),
$$

which gives the lemma because $\mu_{0}\left(x^{n}-t y^{m}\right)=(m-1)(n-1)$.

Proof of Theorem 2.1. It follows from Lemma 4.1 and Theorem 3.1 that for every vector $\vec{v}=(m, n)$, where $m, n$ are co-prime positive integers, the number $l\left(\vec{v}, \mathcal{N}_{J}(f, g)\right)$ depends only on the equisingularity class of the pair $f=0$ and $g=0$. Since every Newton diagram $\Delta$ is equal to the intersection of half-planes determined by $l(\vec{v}, \Delta)$ the theorem is proved.

\section{References}

[1] E. Brieskorn and H. Knörrer, Plane algebraic curves, Birkhäuser, Boston, 1986.

[2] E. Casas-Alvero, Discriminant of a morphism and inverse images of plane curve singularities, Math. Proc. Cambridge Philosophical Society, 135, Cambridge University Press, 2003, 385-394.

[3] E. Casas-Alvero, Jacobian quotients, an algebraic proof, J. Pure Appl. Algebra 208(3) (2007), 1055-1062.

[4] H. Eggers, Polarinvarianten und die Topologie von Kurvensingularitäten: Inauguraldissertation zur Erlangung des Doktorgrades, 147, Math.-Nat.wiss., Fakultät der Universität, Bonn, 1982.

[5] E. García Barroso and J. Gwoździewicz, Characterization of jacobian Newton polygons of plane branches and new criteria of irreducibility, Ann. Inst. Fourier 60(2) (2010), 683-709.

[6] J. Gwoździewicz, A. Lenarcik and A. Płoski, Polar invariants of plane curve singularities: intersection theoretical approach, Demonstratio Math. XLIII(2) (2010), 303-323.

[7] T. Kuo and Y. Lu, On analytic function germs of two complex variables, Topology 16(4) (1977), 299-310.

[8] T. Kuo and A. Parusiński, Newton-Puiseux roots of Jacobian determinants, J. Algebraic Geom. 13(3) (2004), 579-602.

[9] H. Maugendre, Discriminant d'un germe $\Phi:\left(C^{2}, 0\right) \rightarrow\left(C^{2}, 0\right)$ et résolution minimale de $f \cdot g$, Ann. Fac. Sci. Toulouse Math. 7(3) (1998), 497-525.

[10] M. Merle, Invariants polaires des courbes planes, Invent. Math. 41(2) (1977), 103-111.

[11] F. Michel, Jacobian curves for normal complex surfaces, Contemp. Math. 475 (2008), $135-149$.

[12] M. Oka, Non-degenerate complete intersection singularity, Hermann, Paris, 1997.

[13] B. Teissier, The hunting of invariants in the geometry of discriminants, in 'Real and complex singularities Oslo' (P. Holm, ed.), Sijthoff \& Noordhoff Int. Publishers, 1977, 565-677.

[14] B. Teissier, Variétés polaires, Invent. Math. 40(3) (1977), 267-292.

Department of Mathematics, Technical University, AL. 1000 LPP 7, 25-314 Kielce, POLAND

E-mail address: matjg@tu.kielce.pl 